\title{
Terahertz NDE Application for Corrosion Detection and Evaluation under Shuttle Tiles
}

\author{
Robert F. Anastasi ${ }^{1}$, Eric I. Madaras ${ }^{2}$, and Jeffrey P. Seebo ${ }^{3}$, Stephen W. Smith ${ }^{4}$, Janice \\ K. Lomness ${ }^{5}$, Paul E. Hintze ${ }^{5}$, Catherine C. Kammerer ${ }^{6}$, William P. Winfree ${ }^{2}$, and \\ Richard W. Russell ${ }^{7}$ \\ ${ }^{1}$ U.S. Army Research Laboratory, Vehicle Technology Directorate, AMSRD-ARL-VT- \\ SM, Nondestructive Evaluation Sciences Branch, NASA Langley Research Center, MS \\ 231, Hampton, VA 23681 \\ ${ }^{2}$ NASA Langley Research Center, Nondestructive Evaluation Sciences Branch, MS 231 \\ Hampton, VA 23681 \\ ${ }^{3}$ Lockheed Martin, NASA Langley Research Center, MS 231, Hampton, VA 23681 \\ ${ }^{4}$ NASA Langley Research Center, Durability, Damage Tolerance \& Reliability Branch, \\ MS 188E, Hampton, VA 23681 \\ ${ }^{5}$ NASA Kennedy Space Center, Corrosion Technology Laboratory, KT-E, Kennedy \\ Space Center, FL, 32899 \\ ${ }^{6}$ United Space Alliance, NASA Kennedy Space Center, Specialty Engineering and \\ Technical Services, USK-507, Cape Canaveral, FL, 32920-4304 \\ ${ }^{7}$ NASA Kennedy Space Center, NASA Orbiter Project Office, MV7, Kennedy Space \\ Center, FL, 32899
}

\begin{abstract}
Pulsed Terahertz NDE is being examined as a method to inspect for possible corrosion under Space Shuttle Tiles. Other methods such as ultrasonics, infrared, eddy current and microwave technologies have demonstrable shortcomings for tile NDE. This work applies Terahertz NDE, in the frequency range between $50 \mathrm{GHz}$ and $1 \mathrm{THz}$, for the inspection of manufactured corrosion samples. The samples consist of induced corrosion spots that range in diameter $(2.54$ to $15.2 \mathrm{~mm})$ and depth $(0.036$ to $0.787 \mathrm{~mm})$ in an aluminum substrate material covered with tiles. Results of these measurements are presented for known corrosion flaws both covered and uncovered and for blind tests with unknown corrosion flaws covered with attached tiles. The Terahertz NDE system is shown to detect all artificially manufactured corrosion regions under a Shuttle tile with a depth greater than $0.13 \mathrm{~mm}$.
\end{abstract}

Keywords: Terahertz, Nondestructive Evaluation, Corrosion 


\section{INTRODUCTION}

The Space Shuttle is built for space environments and is subjected to extreme mechanical and thermal stress associated with its launch, re-entry into Earth's atmosphere, and landing. Safe and continued operation of the vehicle is of prime importance. All aspects of the vehicle are scrutinized before and after each launch including Shuttle tiles that are inspected for damage and periodically removed for closer examination of the underlying structure. Corrosion is one underlying condition that is evaluated [1] and arises from the inherent use of the vehicle. While these vehicles do not see the number of flights of a commercial aircraft, the unique operating environments experienced by the Shuttles, from a controlled environment, to exposed marine condition at the launch pad, and to the vacuum and harsh environment of space, pose a unique set of conditions. The oxidation of metallic structures in the presence of atmospheric species, oxygen and water vapor can result in a degradation of structural integrity. For aluminum alloys, corrosion generally develops as pitting or thinning and in general changes a nominally smooth surface to an uneven and irregular surface, which can then result in cracking. A potential method for detecting corrosion under tiles is pulsed Terahertz (THz) nondestructive evaluation (NDE). THz electromagnetic waves can penetrate the nonconductive Shuttle tile material and inspect the underlying metallic surface.

Pulsed THz NDE is a relatively new inspection technology $[2,3]$ that offers a non-contact and highresolution inspection method. Its frequency regime is between $300 \mathrm{GHz}$ and $3 \mathrm{THz}$ and it has a free space wavelength range of 1000 to $100 \mu \mathrm{m}$. This is a region of the electromagnetic spectrum between the microwave and infrared bands. Initial applications for $\mathrm{THz}$ electromagnetic waves have focused on spectroscopic applications in astronomy [4], security application for chemical, biological, and weapons detection [5-9], and medical applications [10,11]. Until recently, short pulse sources and detectors were nonexistent in this range, limiting the viability of THz NDE applications. In this paper, the preliminary study of the capabilities of $\mathrm{THz}$ for detecting corrosion through a Shuttle Tile from one side is presented.

The pulsed THz system used in this work has been described in detail elsewhere [12] and was initially designed to inspect up to $30 \mathrm{~cm}$ of Sprayed-On Foam Insulation. The system was integrated with a spatial scanner, data acquisition, and control system that translated the $\mathrm{THz}$ transceiver along two dimensions, and acquired and stored the temporal waveforms at incremental scan points. The system consists of an $800 \mathrm{~nm}$ femtosecond laser source with a pulse rate of $75 \mathrm{MHz}$, a THz control unit with a mechanical scanning optical delay line, a transceiver head with transmitter/receiver, lens for focusing the THz beam, and a computer. Fiber optics connects the laser source to the transceiver and fully contains the laser light, which allows for a freely movable transmitter and receiver. The transceiver for this system, shown in figure 1 , is a transmitter and receiver in a confocal arrangement. This confocal arrangement used a $15 \mathrm{~cm}$ diameter, 285 $\mathrm{mm}$ focal length lens to concentrate the THz energy on the sample to a spot diameter of approximately 3 $\mathrm{mm}$. The digitized time-domain signal is composed of 2048 points and has a duration of $320 \mathrm{ps}$, which corresponds to a propagation path length in air of $96 \mathrm{~mm}$.

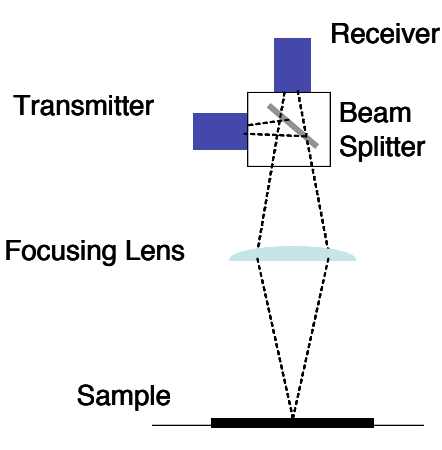

(a)

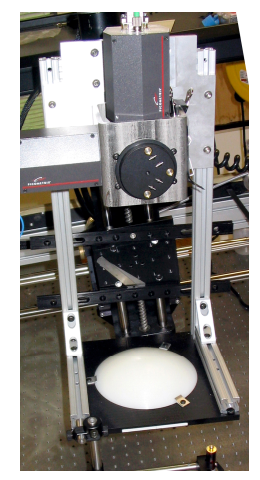

(b)

Figure 1. Transceiver confocal arrangement (a) schematic and (b) photograph. 


\section{TILE RESPONSE TO THz}

To characterize the $\mathrm{THz}$ properties of the tiles, reflections from a metal substrate with and without an intermediary thick, medium, and thin tile were measured. These tiles are shown in figure 2 . The thin tile in figure $2 \mathrm{c}$ was made from an originally thicker tile that was cut. Signals through each tile are shown in figure 3 along with a signal from the bare aluminum plate. These plots show the signal arrival and attenuation as a function of tile thickness. From the signals, the calculated attenuation was $3.06 \mathrm{~dB} / \mathrm{cm}$ at $0.25 \mathrm{THz}$ and the average wave velocity was $2.78 \pm 0.04 \times 10^{8} \mathrm{~m} / \mathrm{s}$. The real part of the refractive index for the material, calculated from the average wave velocity, was $1.08 \pm 0.02$.

\section{CORROSION SAMPLES AND THz IMAGES}

To assess the ability of the $\mathrm{THz}$ system for corrosion detection under Shuttle tiles, tiles were placed on a metal substrate with laboratory-induced corrosion features. In one set of measurements, the corroded areas were covered with detachable tiles to allow for measurements with and without the tiles. In other cases, blind tests were performed on tile samples with defects that were covered by a permanently attached tile. The corrosion specimens were part of panels that were originally test articles for an impact study. These panels consisted of an array of tiles $24.5 \mathrm{~mm}$ thick, attached to an aluminum substrate. Each tile number was used to reference the $\mathrm{THz}$ scans.

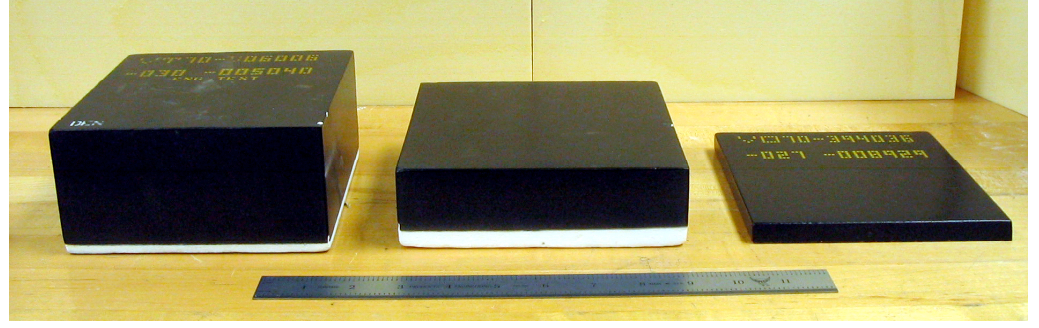

(a)

(c)

Figure 2. Shuttle tiles of various thicknesses: (a) $76 \mathrm{~mm}$, (b) $48 \mathrm{~mm}$, and (c) $12 \mathrm{~mm}$

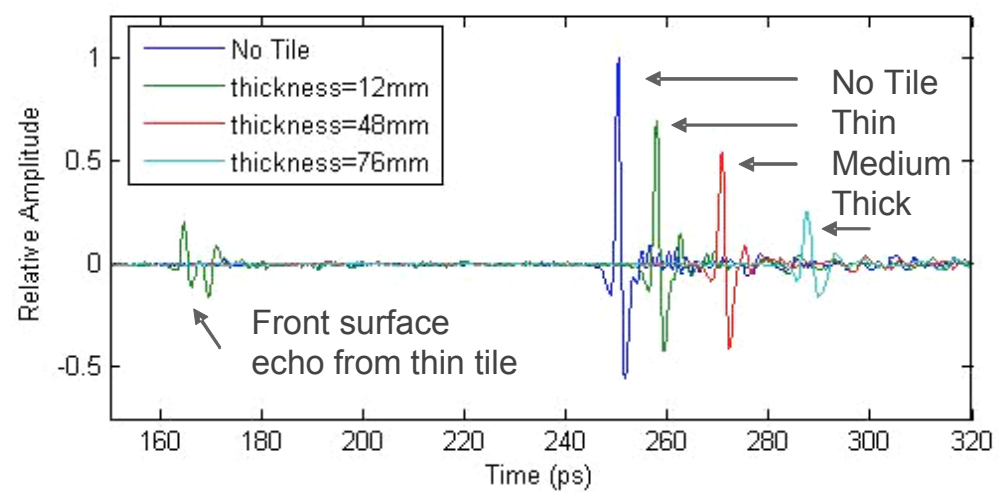

Figure 3. THz response for thick, medium, and thin and tile 
The corroded regions were generated by applying a constant electric current to an electrochemical cell filled with a sodium chloride solution as the electrolyte. The depth of the corrosion was varied by controlling the magnitude and duration of the applied electrical current and the corrosion area was determined by the size of the electrochemical cell. Corrosion depths ranged from 0.08 to $0.51 \mathrm{~mm}$ and were generated with currents between 10 and $30 \mathrm{~mA}$ applied for 2 to 10 hours. Corrosion region diameters ranged from 2.54 to $15.24 \mathrm{~mm}$.

The first test panel is depicted in figure 4a. The number corresponds to the last three digits of the tile identification number and the ' $\mathrm{D}$ ' indicates tiles that were damaged in previous tests. Only numbered tiles were inspected. Two of the tiles were removed and five areas of corrosion were induced for each tile. The shape of the corrosion areas was similar for each tile. One tile was re-bonded to the aluminum substrate and the other tile was not re-bonded. The unbonded tile allowed for inspection of the corrosion damage with and without a tile. Figure $4 \mathrm{~b}$ shows a close-up of the removable tile and its placement position. This tile is numbered '077' in figure $4 \mathrm{a}$ and is shown inverted with the felt pad bonded to it. The location of the rebonded tile was unknown to the personnel conducting the THz study.

The extent of induced corrosion under tile '077', labeled A-E in figure 5a, was determined by producing silicone replicas of the corroded region and examining the replicas using a laser profilometer. Estimates of the maximum depth of corrosion were made. Measured depths and diameters of corrosion regions are given in Table I.

Figure $5 \mathrm{~b}$ shows a THz scan of the bare aluminum surface. The amplitude image is a grey scale representation of the peak-to-peak value at each scan point. The five corrosion spots and some small surface scratches are visible in the image. The tile was placed over the corrosion spots and rescanned. The resulting amplitude image and frequency image at $75 \mathrm{GHz}$ are shown in figure 6. Figure 6a indicates some amplitude variations caused by spatial attenuation variations in the tile and a few darker spot that are larger than the variance related to tile. The contrast between the corrosion spots and the background improves in the frequency image, figure $6 \mathrm{~b}$. The band-pass filtering eliminates some of the background variations that obscure details in the data. The dark spots in figure $6 \mathrm{~b}$ correspond to corroded regions with depths equal to or greater than $0.30 \mathrm{~mm}$.

The other bonded tiles on this first panel were inspected with the THz system. From this inspection, it was determined that tile '043' was the other tile that was removed, due to distinctive spots that were identified. The amplitude and frequency images for this tile had indications similar to those of the corroded regions of tile '077'.

The second panel inspected had 17 tiles with a layout similar to the first panel. Eleven of the 17 tiles were inspected using the THz system. The remaining six tiles were damaged in previous tests. This inspection was a blind test; the personnel inspecting the panel did not know which tile covered induced corrosion. $\mathrm{THz}$ images of only one tile had indications indicative of corrosion. An image of this tile ' 745 ' has distinctive spots that are larger than the tile related variations. This image along with a picture of the 25 induced corrosion spots (obtained after the inspection process) is shown in figure 7. Figure 7a shows the 25 induced corrosion spots and depth estimates made during manufacturing. Figure $7 \mathrm{~b}$ shows the $\mathrm{THz}$ image with eight large obvious corrosion spots and another eight that are smaller and not as evident. Based on the depth measurements and a visual interpretation of the $\mathrm{THz}$ image, the $\mathrm{THz}$ system detected corrosion spot with depths greater than approximately $0.13 \mathrm{~mm}$.

Table I. Corrosion regions depth and diameters for first panel, tile '077'

\begin{tabular}{|c|c|c|}
\hline Region & $\begin{array}{c}\text { Depth } \\
(\mathrm{mm})\end{array}$ & $\begin{array}{c}\text { Diameter } \\
(\mathrm{mm})\end{array}$ \\
\hline $\mathrm{A}$ & 0.06 & 2.54 \\
\hline $\mathrm{B}$ & $<0.06$ & 15.24 \\
\hline $\mathrm{C}$ & 0.95 & 2.54 \\
\hline $\mathrm{D}$ & 0.75 & 15.24 \\
\hline $\mathrm{E}$ & 0.30 & 7.62 \\
\hline
\end{tabular}




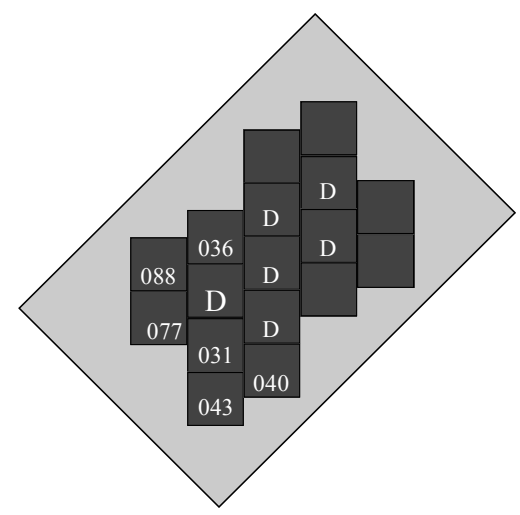

(a)

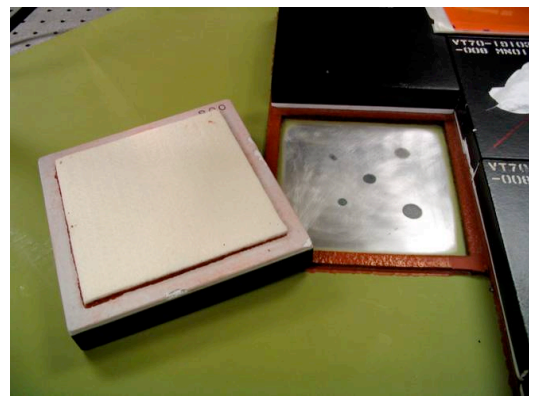

(b)

Figure 4. First test panel (a) schematic and (b) close-up of tile ' 077 ' inverted and induced corrosion areas on metallic substrate. The numbered tiles were inspected and ' $\mathrm{D}$ ' indicates tiles that were damaged in previous tests.

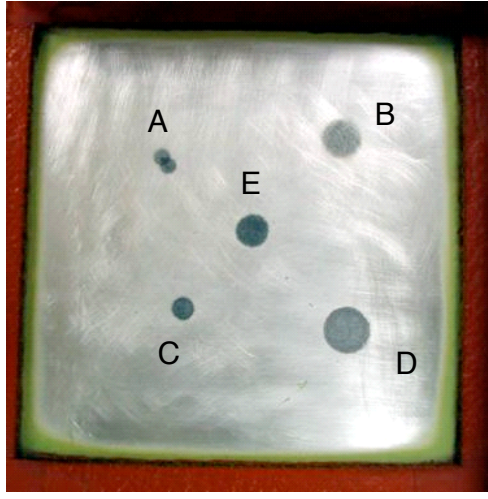

(a)

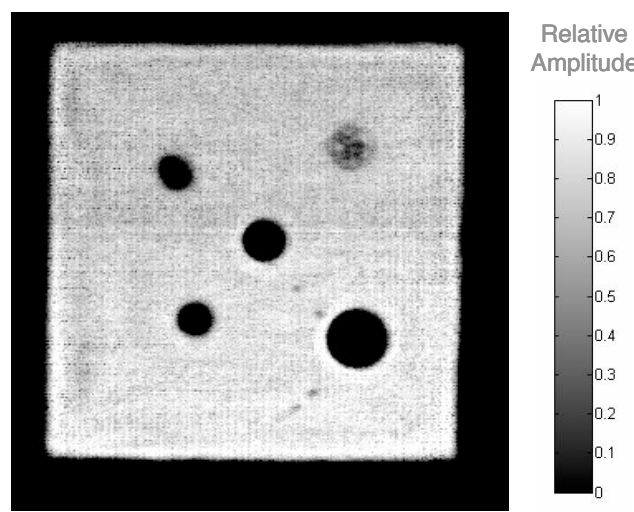

(b)

Figure 5. Induced corrosion sites A-E under tile '077' (a) photograph and (b) THz amplitude image

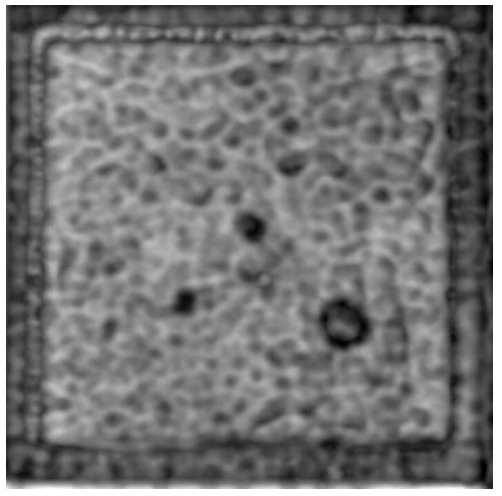

(a)

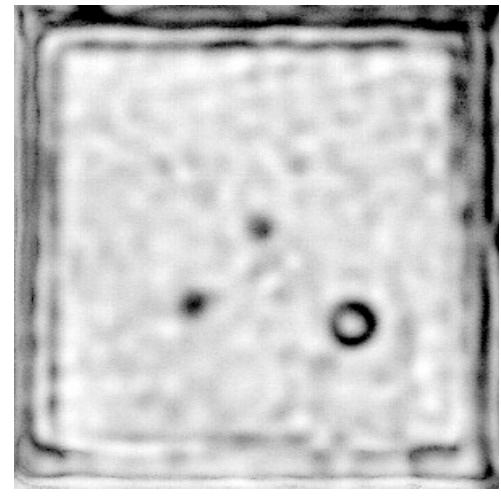

(b)

Figure 6. THz images of induced corrosion covered with tile '077' (a) Amplitude image and (b) Frequency image 


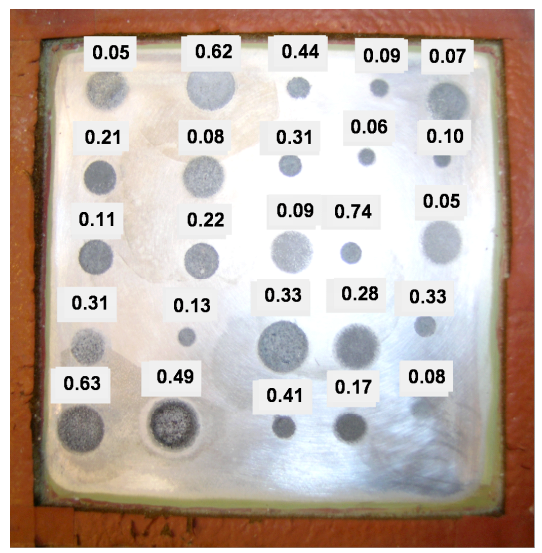

(a)

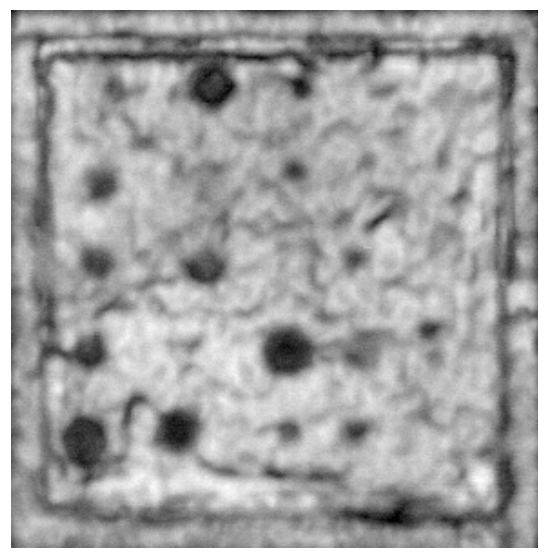

(b)

Figure 7. Panel 2, tile ' 745 ' (a) photograph of induced corrosion spots with estimated depth (mm) at each location and (b) THz amplitude image.

\section{SUMMARY}

Electromagnetic properties of Shuttle Tiles were examined in the $\mathrm{THz}$ regime and found to have a refractive index of 1.08. Inspection of the corrosion panels showed that corrosion on bare aluminum could easily be detected, however when a tile covered the corrosion, the corroded areas were more difficult to detect. This was primarily due to the spatial variation in the $\mathrm{THz}$ properties of the tile and the attenuation of high frequency components of the $\mathrm{THz}$ pulse as it propagates through the tile. Measurements indicate that corroded regions with depths greater than $\sim 0.13 \mathrm{~mm}$ could be detected even when they are covered by a tile. The application reviewed here demonstrates unique capability of THz NDE for detecting hidden corrosion, which was previously considered undetectable when there is only access to one side of the structure.

\section{REFERENCES}

1. Batson, K.W. and J.C. Hess, "Space Shuttle Orbiter Corrosion History," $9^{\text {th }}$ Joint Conference on Aging Aircraft, 2006

2. Mittleman, D., et al., "T-Ray Tomography," Optics Letters, Vol. 22, No. 12, June 1997

3. Dorney, T., et al., "Imaging with Terahertz Pulses," International Symposium on Optical Science and Technology, July 2000

4. Woolard, D.L., E.R. Brown, M. Pepper, and M. Kemp, "Terahertz Frequency Sensing and Imaging: A Time of Reckoning Future Applications," Proceedings of IEEE, Vol. 93, No. 10, pp 1722-1743, (2005)

5. Fitch, M.J., C. Dodson, D.S. Ziomek, and R. Osiander, "Time-Domain Terahertz Spectroscopy of bio-agent Simulants," Chemical and Biological Standoff Detection II, edited by James O. Jensen, Jean-Marc Thériault, Proceedings of SPIE, Vol. 5584, pp 16-22, (2004)

6. Kawase, K., Y. Ogawa, and Y. Watanabe, "Non-Destructive Terahertz Imaging of Illicit Drugs Using Spectral Fingerprints,”Optics Express, Vol. 11, No. 20, pp 2549-2554, (2003)

7. Kemp, M.C., P.F. Taday, B.E. Cole, A.J. Fitzgerald, and W.R. Tribe, "Security Applications of Terahertz Technology," Terahertz for Military and Security Applications, R.J. Hwu, and D.L. Woolard editors, Proceedings of SPIE Vol. 5070, pp 44-52, (2003)

8. Anscombe, N., "No Place to Hide," IEE Review, pp 26-30, (2005)

9. Zimdars, D., J.S. White, G. Stuk, A. Chemovsky, G. Fichter, and S. Williamson, "Large Area High Speed Time Domain $\mathrm{THz}$ Imager for Security and Non-Destructive Evaluation Imaging”THz Technology, Ultrafast Measurements, and Imaging, IEEE, pp 5-6, (2005)

10. Humphreys, K., J. P. Loughran, M. Gradziel, W. Lanigan, T. Ward, J.A. Murphy, C. O’Sullivan, "Medical applications of Terahertz Imaging: a Review of Current Technology and Potential Applications in Biomedical Engineering, " Proceedings of the 26th Annual International Conference of the IEEE EMBS, pp 1302-1305, (2004)

11. Pickwell, E., B. E. Cole, A. J. Fitzgerald, M. Pepper, and V. P. Wallace, "In Vivo Study of Human Skin Using Pulsed Terahertz Radiation,” Phys. Med. Biol., Vol. 49, pp 1595-1607, (2004)

12. Zimdars, D., Valdmanis, J. A., White, J. S., Stuk, G., Williamson, S., Winfree, W.P, and Madaras, E. I., "Technology and Applications of Terahertz Imaging Non-Destructive Examination: Inspection of Space Shuttle Sprayed On Foam Insulation," Review of Progress in Quantitative Nondestructive Evaluation, Golden, Colorado, 25-30 July 2004 